\title{
The Reaction Mechanism with Free Energy Barriers at Constant Potentials for the Oxygen Evolution Reaction at the IrO2 (110) Surface
} Yuan Ping, Robert J. Nielsen, and William A. Goddard

J. Am. Chem. Soc., Just Accepted Manuscript • DOI: 10.1021/jacs.6b07557 • Publication Date (Web): 09 Dec 2016

Downloaded from http://pubs.acs.org on December 13, 2016

\section{Just Accepted}

"Just Accepted" manuscripts have been peer-reviewed and accepted for publication. They are posted online prior to technical editing, formatting for publication and author proofing. The American Chemical Society provides "Just Accepted" as a free service to the research community to expedite the dissemination of scientific material as soon as possible after acceptance. "Just Accepted" manuscripts appear in full in PDF format accompanied by an HTML abstract. "Just Accepted" manuscripts have been fully peer reviewed, but should not be considered the official version of record. They are accessible to all readers and citable by the Digital Object Identifier (DOI®). "Just Accepted" is an optional service offered to authors. Therefore, the "Just Accepted" Web site may not include all articles that will be published in the journal. After a manuscript is technically edited and formatted, it will be removed from the "Just Accepted" Web site and published as an ASAP article. Note that technical editing may introduce minor changes to the manuscript text and/or graphics which could affect content, and all legal disclaimers and ethical guidelines that apply to the journal pertain. ACS cannot be held responsible for errors or consequences arising from the use of information contained in these "Just Accepted" manuscripts. 


\section{Introduction}

Artificial photosynthesis is one of the most promising strategies to convert sunlight to clean fuels ${ }^{1}$. The oxygen evolution reaction (OER) through water oxidation is the most critical step for both water splitting ${ }^{2,3}$ and $\mathrm{CO}_{2}$ reduction ${ }^{4}$. Understanding the atomistic details of the OER mechanism for heterogonous catalysts is extremely important for both interpreting electrochemical measurements and designing new catalysts. Most of the previous theoretical studies of OER catalysts mainly focused on the thermodynamic properties of the reaction intermediates without considering their free energy barriers (including transition states) and kinetic reaction rates in details $^{5-7}$. Indeed most quantum mechanics $(\mathrm{QM})$ calculations have been performed with constant total number of electrons (constant charge), instead of the constant potential conditions of the experiments. The difficulty of QM for treating charged systems in solution has made such constant potential calculations a formidable task until the recent implementation of the constant potential DFT in conjunction with the CANDLE implicit solvent method by Sundararamen et al ${ }^{8}$ and applied recently to $\mathrm{CO}_{2}$ reduction at $\mathrm{Cu}$ surfaces 9 .

$\mathrm{IrO}_{2}$ is the only active OER catalyst that is relatively stable in the acidic condition, which is critical for integration with photoanodes and optimal PEC efficiency ${ }^{10}$. Recently we found that the morphology of $\mathrm{IrO}_{2}$ can modify the catalystphotoanode interfacial energetics dramatically when in contact with water ${ }^{11}$. However, the mechanistic details of $\mathrm{IrO}_{2}$ including kinetic barriers at the constant potential condition have not been reported. Here with $\mathrm{IrO}_{2}$ our prototype, we report the first study of atomistic mechanism for a heterogeneous OER catalyst including free energy barriers at constant potential conditions. We address here: 1) What is the rate determining step (RDS) of the $\mathrm{IrO}_{2}$ OER reaction? 2) How does the constant potential condition affect the reaction barriers compared with the standard constant charge conditions? 3) How does the QM overpotential compare with experiments at a particular electrical current? 4) What does the mechanism suggest for improving OER catalytic efficiency?

The following sections are organized as follows: Computational Methods, Results and Discussions, and Conclusions and Outlooks at the end.

\section{Computational Methods}

We performed first principles calculations of $\mathrm{IrO}_{2}(110)$ surface with plane wave basis sets and GBRV ultrasoft pseudopotentials $^{12}$ within the Quantum-Espresso package ${ }^{13}$. We chose $\mathrm{IrO}_{2}$ (110) surface because it has been shown theoretically to be the most stable surface ${ }^{11}$ similar to other rutile structures, $\mathrm{TiO}_{2}$ and $\mathrm{RuO}_{2}$. We constructed 5 layer slab with inversion-symmetry to avoid net dipoles in the cell (the atomic structure of the $\mathrm{IrO}_{2}$ (110) slab can be found in Fig. S1) and we used $12 \AA$ vacuum to avoid periodic image interactions. We used spin-polarized PBE exchange correlation functional along with the DFT-D2 pair potential dispersion corrections ${ }^{14}$. See SI for further computational details for the Density Functional Theory (DFT) calculations.

We used the Climbing Image-Nudged Elastic Band ${ }^{15}$ (CINEB) method to locate the transition state, as implemented in 
Quantum Espresso. The phonon vibrational frequencies for Gibbs free energy calculations are obtained by Density Functional Perturbation Theory (DFPT) ${ }^{16}$. The vibrational contributions to free energies have been included for both surfaces and molecules. To compute free energy change of elementary reaction steps involving gaseous or liquid molecules, such as water and hydrogen, we took into account the contributions of rotation, translation and vibration to the free molecule, which we obtained from Jaguar package ${ }^{17}$ as well as the solvation energy of water molecule in liquid water $(2.05 \mathrm{kcal} / \mathrm{mol})$. The free energy of gas phase $\mathrm{O}_{2}$ is derived as $\mathrm{G}\left[\mathrm{O}_{2}\right]=$ $4.92(\mathrm{eV})+2 \mathrm{G}\left[\mathrm{H}_{2} \mathrm{O}\right]-2 \mathrm{G}\left[\mathrm{H}_{2}\right]$ by utilizing experimental Gibbs free energy of the reaction $\left(2 \mathrm{H}_{2} \mathrm{O}(\mathrm{l}) \rightarrow \mathrm{O}_{2}(\mathrm{~g})+2 \mathrm{H}_{2}(\mathrm{~g})\right)$ at the standard conditions ${ }^{18}$. See SI for further details of DFPT and Jaguar calculations.

We used the new CANDLE implicit solvation model, which has been shown to perform successfully for various metallic and ionic surfaces ${ }^{19,20}$. We computed the grand free energy at the constant electrochemical potential along with the implicit solvation model, in which the charged surfaces can be effectively screened by the ionic response in solution as implemented in JDFTx ${ }^{8,21,22}$. Computational details of JDFTx can be found in SI.

\section{Results and discussions}

\subsection{Surface Diagram of $\mathrm{IrO}_{2}(110)$}

Before studying the reaction mechanism, it is essential to understand the equilibrium surface structure under OER operating conditions. The $\mathrm{IrO}_{2}$ surface is unstable at basic conditions (the OER efficiency decays significantly after one hour) but recent studies ${ }^{24,25}$ show that it remains stable at $\mathrm{pH}=0$ over several hours. Therefore, we focus on the surface diagram of $\mathrm{IrO}_{2}$ at $\mathrm{pH}=0$ condition.

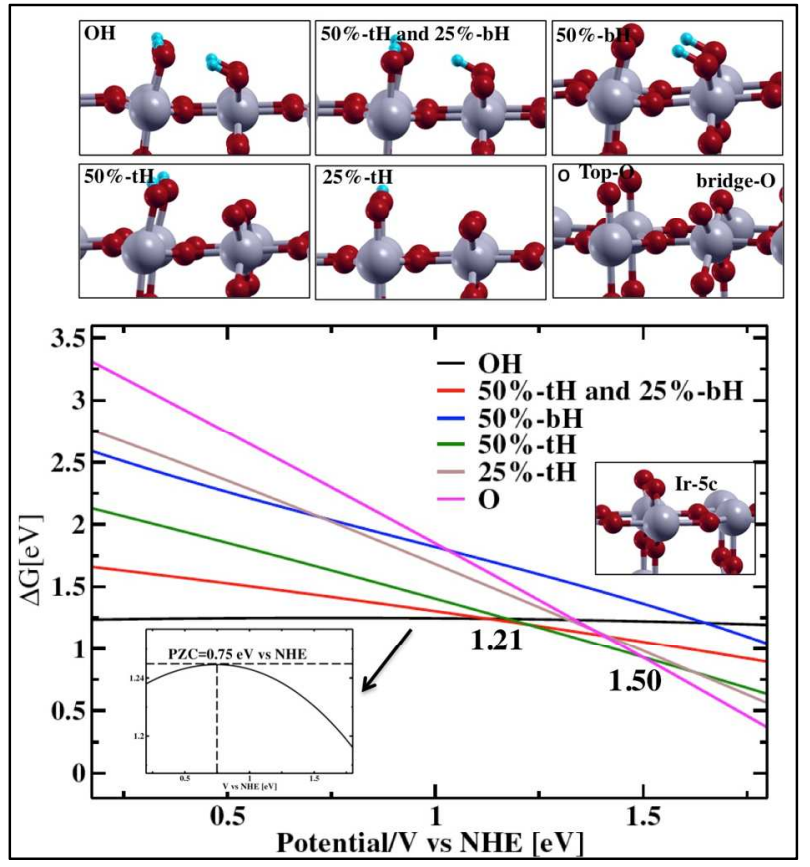

Figure 1. The surface phase diagram of $\mathrm{IrO}_{2}$ (110) with one monolayer of $\mathrm{H}_{2} \mathrm{O}$ as a function of applied potentials. The top 6 panels show the surface structures with different $\mathrm{OH}$ terminations; the bottom panel shows the surface diagram. Black curve: $\mathrm{OH}$ terminated surface; Red curve: $75 \% \mathrm{OH}$ terminated surface with $50 \%$ top $\mathrm{O}$ and
$25 \%$ bridging O; Blue curve: $50 \% \mathrm{OH}$ terminated surface with bridging $\mathrm{O}$; Dark green curve: $50 \% \mathrm{OH}$ terminated surface with top O; Gray curve: $25 \% \mathrm{OH}$ terminated surface with top O; Purple curve: $\mathrm{O}$ terminated surface. The inner figure at the bottom left shows the zoom-in of the black curve ( $\mathrm{OH}$ terminated surface) where the crossing of horizontal tangential line and the vertical line shows the potential of zero charge of $\mathrm{IrO}_{2}$ (110) surface in solution at $\mathrm{pH}=0$. The purple and dark green lines cross at $1.50 \mathrm{eV}$ and the red and dark green lines cross at $1.21 \mathrm{eV}$. The surface structure inside the surface diagram is the stoichiometric (110) $\mathrm{IrO}_{2}$.

For the stoichiometric $\mathrm{IrO}_{2}$ (110) surface, half of the surface Ir atoms are 5 coordinated. We showed that $\mathrm{H}_{2} \mathrm{O}$ binds strongly (by $\sim 1.7 \mathrm{eV} / \mathrm{H}_{2} \mathrm{O}$ in liquid water) at this surface ${ }^{20}$ and the $\mathrm{H}_{2} \mathrm{O}$ molecules spontaneously dissociate to form $-\mathrm{OH}$ at the unsaturated $\mathrm{Ir}(\mathrm{Ir}-5 \mathrm{c})$ and $-\mathrm{OH}$ at the bridging $\mathrm{O}$ (as shown in Fig. 1 "OH" surface structure), independent of the starting surface water configurations (see SI Fig. S2). With increasing the applied potentials, the $\mathrm{OH}$ terminated surface is oxidized to gradually lose $\mathrm{H}$ atoms. We computed the surface free energy as following:

$$
\begin{aligned}
& \Delta G_{\text {surf }}=G_{\text {surf-sol }}-G_{\text {bulk }}-N G_{H 2 O-\text { sol }}+n G_{H+}(p H)+n G_{e}(U) \\
& =G_{\text {surf-sol }}-G_{\text {bulk }}-N G_{H 2 O-\text { sol }}+\frac{n}{2} G_{H 2}(1 a t m, 298 K)+n e(U-N H E)
\end{aligned}
$$

where $\mathrm{G}$ is the grand free energy including $\boldsymbol{N}_{e} \boldsymbol{\mu}_{\boldsymbol{e}}\left(\boldsymbol{N}_{\boldsymbol{e}}\right.$ is the number of electrons at a fixed potential and $\boldsymbol{\mu}_{\boldsymbol{e}}$ is the electron chemical potential); “surf-sol” denotes the solvated (110) $\mathrm{IrO}_{2}$ surface with one layer of explicit $\mathrm{H}_{2} \mathrm{O}$ under applied potentials; "bulk" denotes the pristine bulk $\mathrm{IrO}_{2}$; “ $\mathrm{H}_{2} \mathrm{O}$-sol" denotes solvated water molecule; $\mathrm{N}$ is the number of explicit water molecules in the first water layer; $n$ is the number of $H$ atoms removed from the pristine $\mathrm{IrO}_{2}$ surface plus a layer of water molecules for a particular surface structure. We used the equilibrium relationship at the standard conditions: $\frac{1}{2} G_{H 2}(1 \mathrm{~atm}, 298 K)=G_{H+}(p H=0)+G_{e}(S H E)$ in order to set the reference potentials to that of the Normal Hydrogen Electrode (NHE)[23]. Since we consider $\mathrm{pH}=0$, a free energy correction related to $\mathrm{pH}$ is not needed. Fig. 1 shows that above $1.50 \mathrm{eV}$ (the experimental OER operating potential is around $1.53 \mathrm{eV}$ ), the most stable surface is the $\mathrm{O}$ terminated surface; however, between 1.21 and $1.50 \mathrm{eV}$, the $50 \% \mathrm{OH}$ terminated surface with two $\mathrm{H}$ at the top $\mathrm{O}$ has the lowest surface energy. If instead we use the constant charge condition (as in most previous studies ${ }^{5,6,23}$ ), the free energy of the - OH terminated surface (bare $\mathrm{IrO}_{2}$ surface + one monolayer water) will not depend on the potential (constant at all potentials), because there are no extra electrons exchanging with the reservoir (or $n$ is equal to zero and $\mathrm{G}$ denotes Helmholtz free energy under constant charge condition, independent of electron chemical potential explicitly). However, for the constant potential condition (the experimental condition), the - $\mathrm{OH}$ terminated surface can be charged depending on the applied potential because the grand free energy $G$ explicitly depends on the electron chemical potential as discussed earlier. The potential at which the surface free energy has zero first order derivative with respect to the electron chemical potential is the potential of zero charge of this surface (PZC); which we calculated to be $0.74 \mathrm{eV}$ vs NHE for the - $\mathrm{OH}$ terminated $\mathrm{IrO}_{2}$ (110) surface in water using $\mathrm{PBE}+\mathrm{D} 2$ ). 


\subsection{Thermodynamics of OER reaction}

\subsubsection{Reaction Path at $\mathrm{U}>1.50 \mathrm{eV}$ vs NHE}

As discussed above, at the experimental operating condition $\mathrm{U}>1.50 \mathrm{eV}$ vs NHE, we calculate that the most stable surface structure of $\mathrm{IrO}_{2}(110)$ is the fully $\mathrm{O}$ terminated surface (as shown in Fig.1). We will study the reaction mechanism starting from this surface.

From our spin-polarized PBE+D2 calculations, we found that the most stable $\mathrm{O}$ terminated surface, has 0.86 unpaired spin on the top $\mathrm{O}$ atoms (Lowdin charge; the Ir under the top $\mathrm{O}$ has 0.51 unpaired spin; the bridging $\mathrm{O}$ has close to zero spin), indicating that the top $\mathrm{O}$ atoms have radical character and provide the active sites to form the new $\mathrm{O}-\mathrm{O}$ bonds that are essential for $\mathrm{O}_{2}$ production. Next we performed detailed calculations to validate this expectation.

In order to form an $\mathrm{O}-\mathrm{O}$ bond at the $\mathrm{IrO}_{2}(110) \mathrm{O}$ terminated surface, there are two possible mechanisms: 1 . two neighboring $\mathrm{O}$ atoms couple and form a new $\mathrm{O}-\mathrm{O}$ bond; 2. a solvent water molecule attacks the surface $\mathrm{O}$ to form an $\mathrm{O}-\mathrm{O}$ bond.

For mechanism 1, we found that O-O coupling between a top $\mathrm{O}$ and a bridging $\mathrm{O}$ is unstable (the $\mathrm{O}-\mathrm{O}$ bond breaks apart during geometry optimization), while it is thermodynamically unfavorable between two neighboring top $\mathrm{O}$ atoms (the formation of O-O bond costs $0.62 \mathrm{eV}$ free energy (detailed structure can be found in SI, Fig. S6), which costs much more energy compared to the reaction intermediates in mechanism 2 as shown later.) Therefore, we focus on the mechanism 2 involving an aqueous water attacking the surface $\mathrm{O}$ and dissociating.
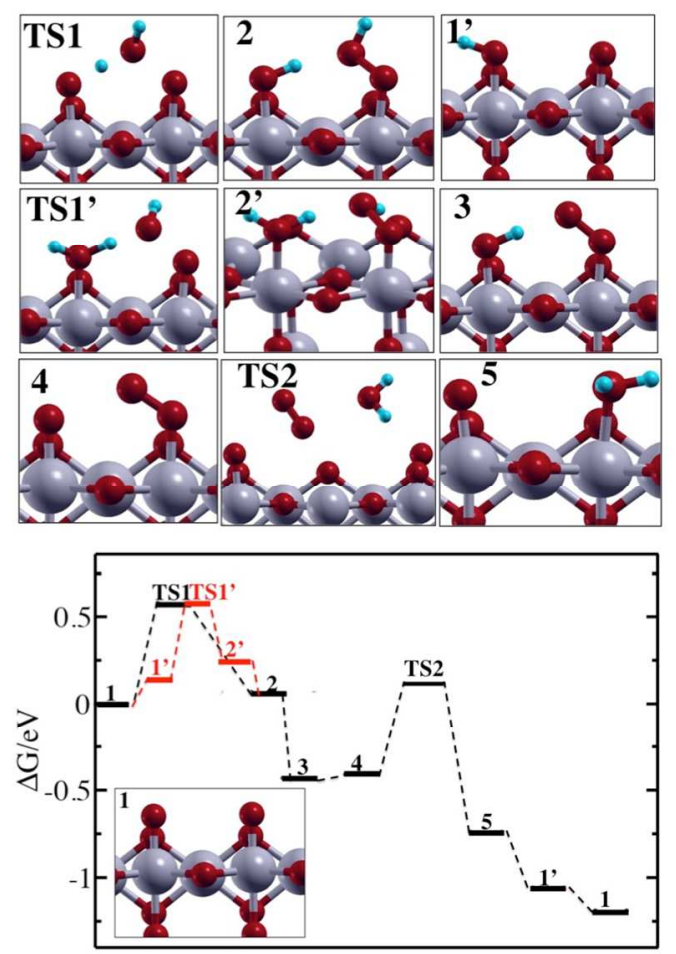

Figure 2. The optimized structure and free energy profile of intermediates and transition states of water dissociation reaction at $\mathrm{U}=1.53$ eV vs NHE. Red balls are O; blue balls are H; silver balls are Ir.

We compared the free energy of all possible positions of dissociated $\mathrm{H}_{2} \mathrm{O}$ and found both $-\mathrm{OOH}$ and $-\mathrm{OH}$ bonds prefer to form at the top $\mathrm{O}$ atoms $(0.21 \mathrm{eV}$ more favorable than $-\mathrm{OOH}$ at the top $\mathrm{O}$ and $-\mathrm{OH}$ at the bridging $\mathrm{O} ; 0.51 \mathrm{eV}$ more favorable than $-\mathrm{OOH}$ at the bridging $\mathrm{O},-\mathrm{OH}$ at the top $\mathrm{O} ; 0.86 \mathrm{eV}$ more favorable than both $-\mathrm{OOH}$ and $-\mathrm{OH}$ at the bridging $\mathrm{O}$.) This is consistent with the indication of radical characters at the top $\mathrm{O}$ atoms at $\mathrm{O}$ terminated $(110) \mathrm{IrO}_{2}$ surface as discussed above, which is more favorable than usual for the site to form a new bond to other atoms.

Fig. 2 shows the reaction intermediates and transition state structures as well as their free energy profile computed at the constant potential condition (more details can be found in SI, Table S1). The difference of free energy barriers and reaction energies between at the constant charge and constant potential conditions varies as a function of applied potential (details see SI, Table S1). Most importantly, at the constant potential condition, the steps that are electrochemically independent at the constant charge condition become dependent on the electrochemical potential. For example, the first water dissociation step (shown in Fig. 2 reaction $1 \rightarrow 2$ ) depends strongly on the electrochemical potential (as shown in Fig. 3) with a slope of 0.5 , which indicates that 0.5 electrons are depleted from the $\mathrm{IrO}_{2}$ surface in this process. This is completely neglected in the constant charge calculation - in fact, we found that at the constant charge condition the work function decreases by $1 \mathrm{eV}$ after one water molecule dissociates at the $\mathrm{O}$ terminated surface (or the Fermi level of the surface is $1 \mathrm{eV}$ closer to the vacuum, details see SI-Fig.S3); therefore, the catalyst $\mathrm{IrO}_{2}$ surface needs to lose electrons to the electrode in order to keep the Fermi level constant before and after the water dissociation at the surface. Moreover, the large variation of work function during the water dissociation reaction process at the constant charge condition indicates the possible large discrepancy from the experimental constant potential condition. In addition, we note that the free energy of the transition states (between two fixed intermediates) also follow the linear dependence with applied potentials (Details see SI-Fig.S5).

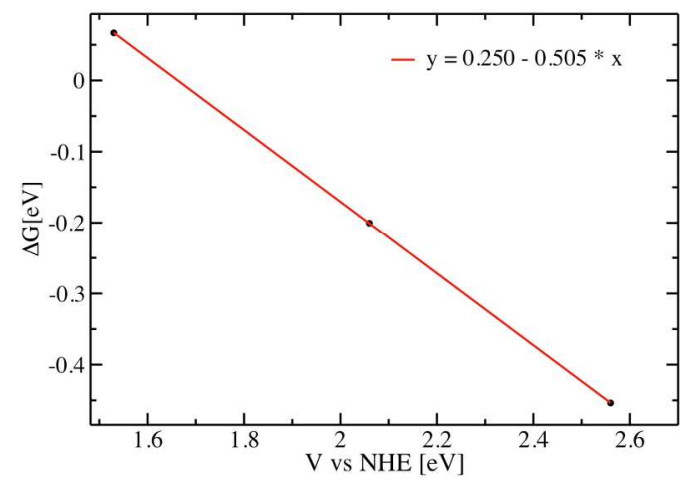

Figure 3. The free energy change of the water dissociation step (reaction $1 \rightarrow 2$ in Fig. 2) as a function of the applied potential vs NHE by the constant potential calculations; black dots: calculated; red line: linear regression of black dots.

For an external potential of $\mathrm{U}=1.53 \mathrm{eV}$, we found that all reaction steps are exothermic (the black reaction path), showing 
that OER is favorable at this potential (consistent with the experimental observation ${ }^{24,25}$ ). Specifically, after water dissociated at the top $\mathrm{O}$ atoms, surface $-\mathrm{OOH}$ that is formed can then lose its $\mathrm{H}$ barrierlessly to form $-\mathrm{OO}^{-}$(as shown in Fig. 4) with bond length $1.28 \AA$. One evidence showing this step is barrierless is that with a second layer of explicit $\mathrm{H}_{2} \mathrm{O}$ molecules on top of surface we found that the -OOH at structure 2 in Fig.2, will spontaneously lose $\mathrm{H}$ to an explicit $\mathrm{H}_{2} \mathrm{O}$ molecule then form $\mathrm{H}_{3} \mathrm{O}^{+}$and $-\mathrm{OO}^{-}$at the $\mathrm{IrO}_{2}$ surface (during geometry optimizations.) Fig. 4 shows one unpaired electron distributed at the $\mathrm{O}-\mathrm{O} \pi^{*}$ orbital.

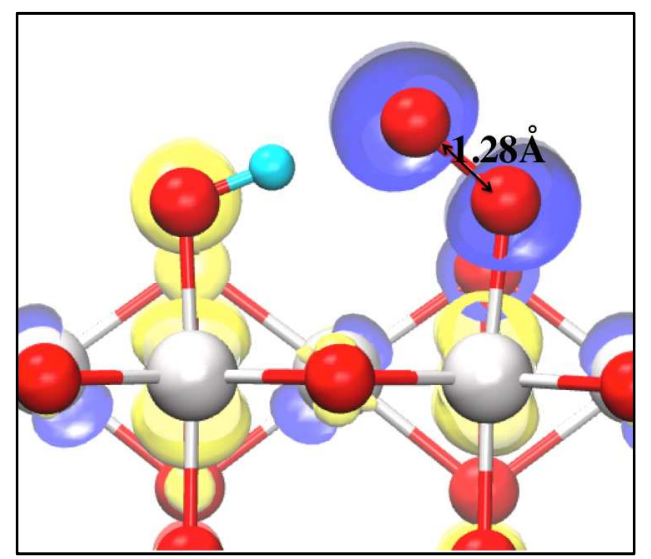

Figure 4. Spin density distribution (spin up-spin down) for surface structure 3 (blue is negative sign; yellow is positive sign); red balls are $\mathrm{O}$; blue balls are $\mathrm{H}$; silver balls are Ir.

At this high potential (1.53 eV vs NHE) the surface $\mathrm{H}$ atoms are removed so that a free $\mathrm{OO}^{-}$will evolve from the surface to form $\mathrm{O}_{2}$ molecule while leaving behind an $\mathrm{O}$ vacancy. (We found that without the neighbor $\mathrm{H}$, the free energy to remove $\mathrm{O}_{2}$ from the surface is decreased by $0.2 \mathrm{eV}$ because the interaction between surface $\mathrm{H}$ and $\mathrm{O}_{2}$ is absent). Meanwhile, a second aqueous $\mathrm{H}_{2} \mathrm{O}$ molecule can bind to the $\mathrm{O}$ vacancy site. This leads to an extraordinarily large $0.94 \mathrm{eV} \mathrm{H}_{2} \mathrm{O}$ binding energy to the $\mathrm{O}$ vacancy site. In a previous study we also found that the $\mathrm{H}_{2} \mathrm{O}$ binding energy at the bare $\mathrm{IrO}_{2}(110)$ surface is nearly twice that of the bare $\mathrm{TiO}_{2}(110)$ surface ${ }^{20}$. After $\mathrm{H}_{2} \mathrm{O}$ attaches to the vacancy, surface (structure 5 in Fig. 2) will be deprotonated to reform the stable $\mathrm{O}$ terminated surface.

An alternative path (the red path as shown in Fig. 2) is to first protonate the $\mathrm{O}$ terminated surface at the top $\mathrm{O}$ atom (to form $25 \% \mathrm{OH}$ terminated surface) then allow an aqueous water molecule to dissociate at the surface. This path is thermodynamically less favorable by $0.13 \mathrm{eV}$ compared with the direct water dissociation path (black path in Fig. 2). Interestingly, this second path has a lower reaction barrier by $0.13 \mathrm{eV}$ compared to the direct path, making it kinetically more favorable. (Reactions 1->1' and 2' $->2$ are barrierless and kinetically fast processes, similar to what we discussed above). This result conveys an important message here: the reaction barriers need not be proportional to the reaction energies of the intermediates, a simple concept often assumed to be fundamental in many previous studies of OER mechanism at oxide surfaces, where only the free energies of the intermediates were computed $^{3,26,27}$.

\subsubsection{Reaction Path at $1.21<\mathrm{U}<1.50 \mathrm{eV}$ vs NHE}
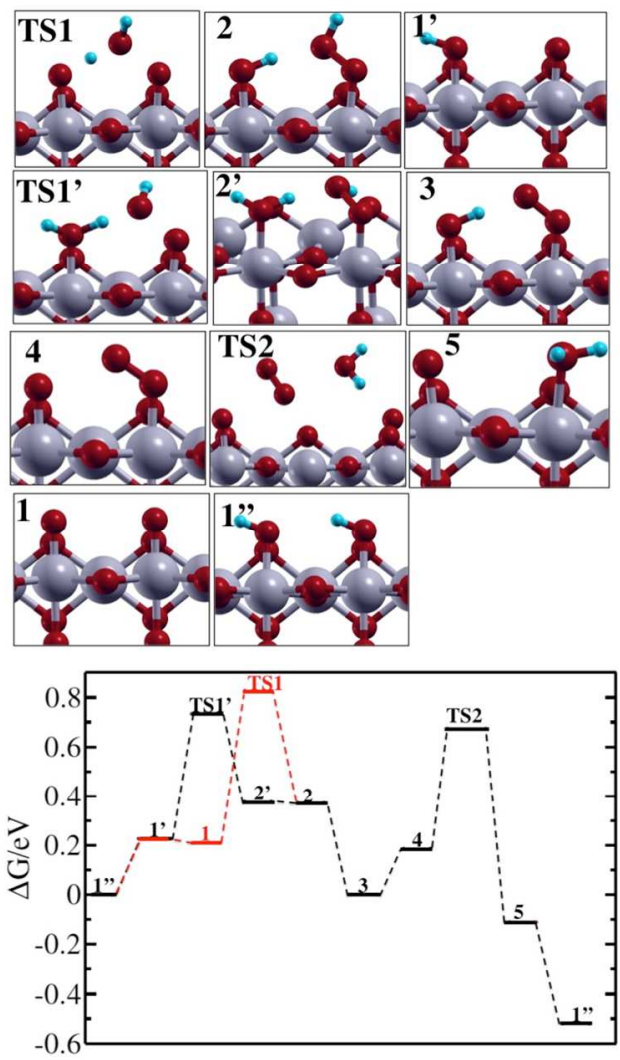

Figure 5. The optimized structure and free energy profile of reaction intermediates and transition states of water dissociation reaction at $\mathrm{U}=1.36 \mathrm{eV}$ vs NHE. Red balls are $\mathrm{O}$; blue balls are $\mathrm{H}$; silver balls are Ir.

To complete our mechanistic study, we also investigated the reaction mechanism at the potential less than $1.50 \mathrm{eV}(1.36 \mathrm{eV}$ vs NHE), although the experimental potential is larger than 1.5 $\mathrm{eV}$. At this potential, the most stable surface structure is $50 \%$ $\mathrm{OH}$ terminated surface at the top $\mathrm{O}$ atoms (surface structure 1" in Fig. 5). After the top $\mathrm{O}$ attaches an $\mathrm{H}$ atom to form $\mathrm{OH}$, no unpaired spin is left so that the top $\mathrm{O}$ atoms are not reactive anymore. Therefore, in order to form the $-\mathrm{OO}$ bond at the surface, we must remove at least one $\mathrm{H}$ atom from the top $\mathrm{O}$ atoms as shown in Fig.5 (surface structure 1 and 1').

The black line in Fig.5 shows the reaction path with one surface $\mathrm{H}$ removed from 1" before the $\mathrm{H}_{2} \mathrm{O}$ dissociation step; the red line shows both surface $\mathrm{H}$ atoms removed from 1 " before $\mathrm{H}_{2} \mathrm{O}$ dissociation. For both reaction paths, most intermediates have reaction free energies that are endothermic indicating that at the potential $1.36 \mathrm{eV}(<1.50 \mathrm{eV})$, OER may be slow at the $\mathrm{IrO}_{2}(110)$ surface. Another important result here is, that as in the case for $\mathrm{U}>1.50 \mathrm{eV}$, the reaction path with slightly more stable intermediates (reaction $1 \rightarrow 2$ compared to $1{ }^{\prime} \rightarrow 2$ ' ; details see SI, Table S1), has higher reaction barriers (TS1 compared to TS1'). Again this shows that the reaction steps that are thermodynamically more favorable can be kinetically less favorable.

\subsection{Transition States of OER}

\subsubsection{Transition States for Water Dissociation}




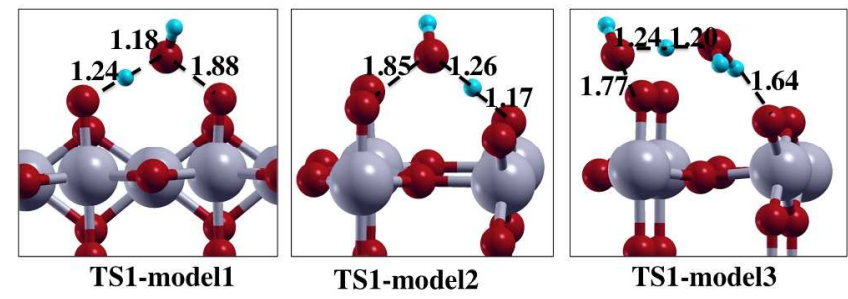

Figure 6. Transition states of one water dissociated at the O terminated surface. "TS1-model1" is the transition state of both $-\mathrm{OOH}$ and -OH at top O; "TS1-model2" is the transition state of $-\mathrm{OOH}$ at the top $\mathrm{O}$ and $-\mathrm{OH}$ at the bridging $\mathrm{O}$; "TS1-model3" is the transition state of $-\mathrm{OOH}$ at the top $\mathrm{O}$ and $-\mathrm{OH}$ at the bridging $\mathrm{O}$ with two explicit water molecules instead of one.

Next we examine the important transition states in detail (other reaction steps are barrierless). We computed all possible transition state structures of one $\mathrm{H}_{2} \mathrm{O}$ molecule dissociated at the $\mathrm{IrO}_{2}(110) \mathrm{O}$ terminated surface as shown in Fig.6. We found although the final state of the "TS1-model2" is $0.2 \mathrm{eV}$ higher than the "TS1-model1", the reaction barriers from the reactants to the transition states are very similar (difference of $10 \mathrm{meV}$ ). In order to understand the effect of more explicit water molecules, we added a second water molecule and reexamined the transition state barriers: we found "TS1-model3" has a barrier of $0.61 \mathrm{eV}$ (similar to the "TS1-model1" $0.58 \mathrm{eV}$ and the "TS1-model2" $0.59 \mathrm{eV}$, when all the transition states referenced to the initial state of $\mathrm{O}$ terminated surface plus one or two $\mathrm{H}_{2} \mathrm{O}$ molecules from the bulk water) at $\mathrm{U}=1.53 \mathrm{eV}$ vs NHE. We note that we compared the stability of different water configurations for two $\mathrm{H}_{2} \mathrm{O}$ at $\mathrm{IrO}_{2}(110)$ surface and the initial state for “TS1-model3" gives $0.5-0.7 \mathrm{eV}$ lower energy compared with other configurations (see SI-Fig. S4 for details). Interestingly, the transition state with two explicit water molecules involves a proton transfer between two water molecules as shown in Fig. 6. These results confirm that the reaction barrier of $\mathrm{H}_{2} \mathrm{O}$ dissociation is insensitive to the explicit $\mathrm{H}_{2} \mathrm{O}$ configuration and number of explicit water molecules in the calculations.

\subsubsection{Transition States of $\mathrm{O}_{2}$ Removal}

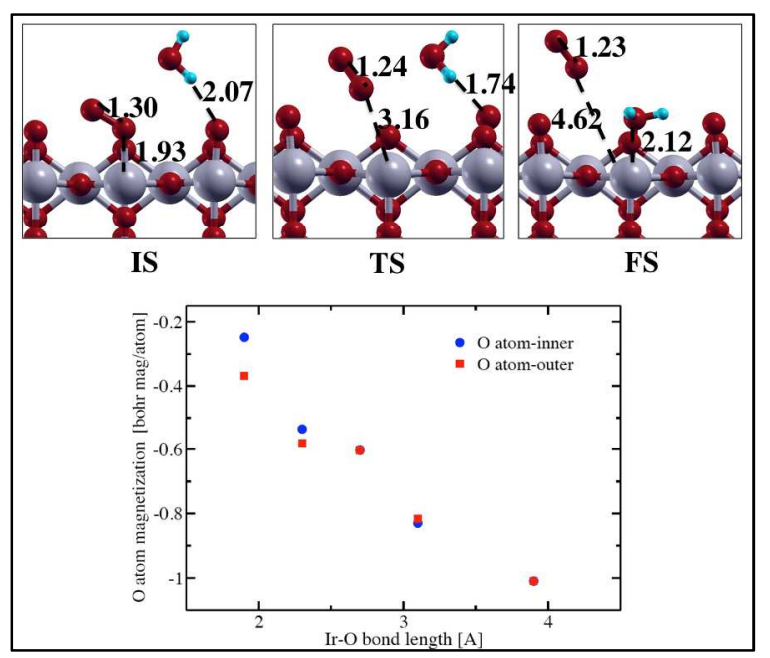

Figure 7. Top: initial, transition state and final structures of the process of one $\mathrm{O}_{2}$ substitute by a water molecule. Bottom: the magnetization of two $\mathrm{O}$ atoms (blue dot: the $\mathrm{O}$ atom attached to the surface; red square: the $\mathrm{O}$ atom pointing away from the surface) as a function of Ir-O bond length.

Removing $\mathrm{O}_{2}$ from the surface is a non-trivial step involving several complications: 1) it is unclear whether $\mathrm{O}_{2}$ dissociation from the surface and $\mathrm{H}_{2} \mathrm{O}$ binding to the $\mathrm{O}$ vacancy is simultaneous or sequential; 2) whether DFT can capture the $\mathrm{O}_{2}$ spin changes from doublet $\mathrm{O}_{2}{ }^{-}$at the surface to triplet $\mathrm{O}_{2}$ molecule in solution or air; 3) we must consider how to take into account the change of $\mathrm{O}_{2}$ free energy from the oxide surface to solution or vacuum. We computed the energy barrier of one $\mathrm{O}_{2}$ substituted by one $\mathrm{H}_{2} \mathrm{O}$ molecule using CI-NEB at the $\mathrm{PBE}+\mathrm{D} 2$ level of theory (structures of the initial state, transition state and final state are shown in Fig.7) We found an energy barrier of $0.29 \mathrm{eV}$ (if the translational and rotational enthalpy and entropy of $\mathrm{O}_{2}$ is from the gas phase, computed by Jaguar) and $0.46 \mathrm{eV}$ (if we consider the translational and rotational enthalpy and entropy of $\mathrm{O}_{2}$ is from $1 \mathrm{M} \mathrm{O}_{2}$ in liquid $\mathrm{H}_{2} \mathrm{O}$, with $0.17 \mathrm{eV}$ difference from the gas phase, computed by the Henry' s law: $\Delta G=-R T \ln K_{H}$ ) at $\mathrm{U}=1.53 \mathrm{eV}$ vs NHE.

The initial structure has an Ir-O bond length $1.93 \AA$ while the transition state has an Ir-O bond length $3.16 \AA$, thus the explicit water molecule did not participate in the reaction. Instead it has only a van der Waals contact to the surface; which the energy goes down by $0.94 \mathrm{eV}$ after $\mathrm{H}_{2} \mathrm{O}$ comes in and binds to the $\mathrm{O}$ vacancy site. (The NEB path is included in the SI, Fig. S7). This is consistent with the hydrophobic character of the $\mathrm{O}_{2}$ molecule (or low solubility of $\mathrm{O}_{2}$ in $\mathrm{H}_{2} \mathrm{O}$ ). Therefore the $\mathrm{H}_{2} \mathrm{O}$ does not interact with the $\mathrm{O}_{2}$ molecule and instead binds to the surface vacancy site after the $\mathrm{O}_{2}$ is sufficiently far away (4.62 $\AA$ ) from the surface.

As discussed above (and shown in Fig. 4), $\mathrm{O}_{2}$ has one unpaired spin when attached to $\mathrm{IrO}_{2}$ surface. We traced the change of the spins on the two $\mathrm{O}$ atoms (computed as Lowdin charges and renormalized by the atomic spin of an isolated $\mathrm{O}_{2}$ molecule, also computed using the Lowdin charge, in order to remove the error of the absolute values from Lowdin charge) during the process of removing $\mathrm{O}_{2}$ from the surface as a function of Ir-O atom distance (as shown in the bottom figure of Fig. 7). As the Ir-O distance increases from 1.93 to $4 \AA$, the atomic spin of one $\mathrm{O}$ atom increases from $\sim 0.3$ to $\sim 1.0$ a.u. indicating a spin doublet to triplet transition for $\mathrm{O}_{2}$. Although the PBE+D2 level of theory cannot describe the energy difference of different $\mathrm{O}_{2}$ electronic states accurately (requiring multi-determinants beyond density function theory to accurately reproduce the difference between the ground triplet state and first excited state), the net spin on the $\mathrm{O}_{2}$ is described fairly well.

The remaining question is what is the rate-determining step (RDS) for the OER reaction at the $\mathrm{IrO}_{2}(110)$ surface? We found that the water dissociation step has the highest free energy barrier which demonstrates this is the rate determining step, consistent with the previous results on similar surfaces such as $\mathrm{RuO}_{2}$ (110) in $\mathrm{Ref}[5]$. We note that although the barrier of $\mathrm{O}_{2}$ removing step is fairly high (about $0.1 \mathrm{eV}$ lower barrier than the water dissociation step), we found the $\mathrm{O}_{2}$ may be overbinded due to the limitation of semi-local functionals (more detailed discussions can be found in SI- Fig.S8). 


\subsection{Micro-kinetic Model and Comparison With the Exper- imental Tafel Line}

An important advantage of performing calculations at the constant potential condition is that the relationship between the activation energy barrier and the electrochemical potential can be obtained naturally with the surface charges adjusted by the constant potential, with no need to manually introduce charges to the surface as done in Ref.18. With the reaction barriers as a function of electrochemical potentials, we can obtain the reaction rates and the currents based on the classical Transition State Theory.

In previous studies, only a single reaction barrier has been assumed to be rate determining in predicting reaction rates; however, our study finds two possible reaction paths can coexist at both low $(<1.5 \mathrm{eV})$ and high $(>1.5 \mathrm{eV})$ potentials. Therefore, we considered a more sophisticated micro-kinetic model to obtain our reaction rates by (More detailed derivation can be found in SI):

$R=k_{25}\left[C_{25}\right]+k_{0}\left[C_{0}\right](2)$

where $\mathrm{R}$ is the reaction rate, $k_{25}=A \exp \left(-\frac{G_{25}(\eta)}{k T}\right)$ based on the transition state theory, $\mathrm{A}$ is $\frac{k T}{h}=6.25 \times 10^{12}$ at the standard condition $298 \mathrm{~K}$ and $1 \mathrm{~atm}, \mathrm{G}_{25}$ is the reaction barrier of the water dissociated at the $25 \% \mathrm{OH}$ covered surface as a function of overpotential $\eta$ (defined as the potential relative to $1.23 \mathrm{eV}$ plus NHE); $\mathrm{k}_{25}$ is the reaction rate for this particular reaction at one active site; $\left[\mathrm{C}_{25}\right]$ is the concentration of active sites at $25 \%$ $\mathrm{OH}$ terminated surface. The notations for the reaction of water dissociation at the $\mathrm{O}$ terminated surfaces $(0 \% \mathrm{OH}$ coverage) $\mathrm{k}_{0}, \mathrm{c}_{0}$ are analogous. We note that we only considered the reaction rates at $25 \% \mathrm{OH}$ covered surface and $\mathrm{O}$ terminated surfaces because water only dissociates at these two surfaces from our calculations.

We can obtain the concentrations of $\left[\mathrm{C}_{25}\right]$ and $\left[\mathrm{C}_{0}\right]$ from the equilibrium constants between surfaces with different $\mathrm{OH}$ coverage concentrations:

$\left[M_{0}\right]=\left[C_{0}\right]+\left[C_{25}\right]+\left[C_{50}\right]+\left[C_{75}\right]+\left[C_{100}\right](3)$

where $\left[M_{0}\right]$ is the total concentration of surface active sites, which is a sum of the active sites at different $\mathrm{OH}$ coverage. $\left[M_{0}\right]$ can be computed by active sites divided by surface area. The concentrations of $\left[C_{0}\right]$ and $\left[C_{25}\right]$ can be written by $\left[M_{0}\right]$ based on Eq. 3 and their surface equilibrium constants:

$$
\left[C_{0}\right]=\frac{\left[M_{0}\right]}{1+K_{025}+K_{050}+K_{075}+K_{0100}}
$$

where $\boldsymbol{K}_{\mathbf{0 2 5}}=\boldsymbol{C}_{\mathbf{2 5}} / \boldsymbol{C}_{\mathbf{0}}$ is the equilibrium constant between $25 \% \mathrm{OH}$ covered surface and $\mathrm{O}$ terminated surface which can be computed by $\boldsymbol{K}_{\mathbf{0 2 5}}=\boldsymbol{A} \boldsymbol{\operatorname { x x p }}\left(-\frac{\boldsymbol{G}_{\mathbf{0 2 5}}(\boldsymbol{\eta})}{\boldsymbol{k} \boldsymbol{T}}\right)$, analogous to $k_{25}$ we discussed above. We can also express $\left[\boldsymbol{C}_{\mathbf{2 5}}\right]$ similarly by combining Eq. 4 and $\boldsymbol{K}_{\mathbf{0 2 5}}=\boldsymbol{C}_{\mathbf{2 5}} / \boldsymbol{C}_{\mathbf{0}}$. Afterward we obtain the overall reaction rates by:

$$
R=\frac{\left[M_{0}\right] k_{0}+\left[M_{0}\right] k_{25} K_{025}}{1+K_{025}+K_{050}+K_{075}+K_{0100}}
$$

Finally we convert reaction rate $\mathrm{R}$ to currents in order to compare with experimental electrochemical measurements:

$\ln j(\eta)=\ln \left(n F N_{A}^{-1} R(\eta)\right)(6)$

where $n$ is the charge transfer in OER reaction; $\mathrm{F}$ is the Faraday constant and $\mathrm{N}_{\mathrm{A}}$ is the Avogadro constant. Fig. 8 shows our calculated relationship between $\ln j$ and overpotential $\eta$ the Tafel plot.

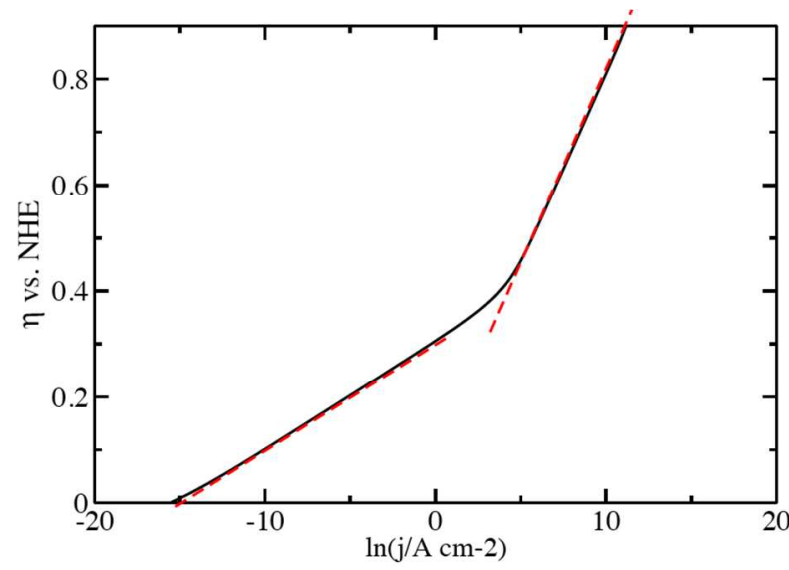

Figure 8. The calculated Tafel lines $(\ln (\mathrm{j})$ vs overpotential for OER on $\mathrm{IrO}_{2}(110)$ (black lines). The red dotted lines show the linear regions in the Tafel plot, which cross at 0.3 volts. Above $0.35 \mathrm{~V}$, the Tafel slope is $73 \mathrm{meV}$; below $0.3 \mathrm{~V}$, the Tafel slope is $22 \mathrm{meV}$.

There are two linear region before and after $\sim 0.3 \mathrm{~V}$. Above $0.35 \mathrm{~V}$, the Tafel slope is $73 \mathrm{meV}$, which is in good agreement with the experimental Tafel slope of $61-85 \mathrm{meV}$ at the overpotential of $0.32-0.47 \mathrm{eV}^{28}$. Below $0.30 \mathrm{~V}$, we found the Tafel slope to be $22 \mathrm{meV}$, which indicates that a different reaction dominates the reaction rates at the lower potential compared to higher potential, as discussed above. The overpotential corresponding to the current $\mathrm{j} 10 \mathrm{~mA} / \mathrm{cm}^{2}$ ( $\operatorname{lnj}$ is -4.6$)$ is $0.22 \mathrm{~V}$, which is in good agreement with the experimental overpotential of $0.27 \pm 0.03 \mathrm{~V}^{24}$. The excellent agreement with the experimental electrochemical measurements demonstrate that our reaction barrier calculations and micro-kinetic models quantitatively explain the OER reaction mechanism at $\mathrm{IrO}_{2}$ (110) surface.

\section{Conclusions and Outlook}

In this work we used DFT Quantum Mechanics in the first investigation of the OER reaction mechanism at the $\mathrm{IrO}_{2}(110)$ surface including kinetic barriers at constant potential conditions. The computed Tafel slope and overpotentials based on the micro-kinetic model are in excellent agreement with experimental electrochemical measurements. Our major findings are:

1) the rate-determining water dissociation step is electrochemically dependent at the constant potential condition (although not at the constant charge condition); 
2) the reaction steps that are thermodynamically favorable are in some cases kinetically less favorable as discussed in section 3.2 ;

3) $\mathrm{O}_{2}$ dissociation from the surface goes continuously from a doublet state when bound to the surface to a triplet state at the dissociated state, and $\mathrm{H}_{2} \mathrm{O}$ does not bind to the $\mathrm{O}$ vacancy site until $\mathrm{O}_{2}$ is removed from the surface.

The observation that the active sites are the top $\mathrm{O}$ atoms with unpaired spins indicates the surface orientation with the maximum number of unsaturated Ir atoms may be the most active. This is because unsaturated Ir atoms on the bare surface will react with $\mathrm{H}_{2} \mathrm{O}$, to from surface $\mathrm{OH}$ sites due to the large water binding energy at $\mathrm{IrO}_{2}$ surface, which leads to radical character on the surface $\mathrm{O}$ after deprotonation at the OER operating potential. This suggests that the less stable (100) $\mathrm{IrO}_{2}$ surface may be more active than the (110) $\mathrm{IrO}_{2}$ surface as discussed in Ref 28.

The insights from our mechanistic study of $\mathrm{IrO}_{2}$ (110) surface OER reaction should provide guidance for designing more active catalysts.

\section{ASSOCIATED CONTENT}

\section{Supporting Information}

Computational details of DFT calculations performed by Quantum Espresso, JDFTx, Jaguar software. Reaction energies and barriers at constant potential and constant charge conditions. Surface structure of the $\mathrm{O}-\mathrm{O}$ coupling intermediate at the $\mathrm{IrO}_{2}(110)$ surface, other initial geometries for a layer of explicit water at pristine (110) $\mathrm{IrO}_{2}$ surface, other geometries for the intial states of TS-model 3. Work functions of $\mathrm{IrO}_{2}$ surface during water dissociation reaction. Linearity of Reaction Barrier Free Energy as a function of the applied potential. CI-NEB path of $\mathrm{O}_{2}$ removing from $\mathrm{IrO}_{2}$ surface. Details of the microkinetic models. $\mathrm{O}_{2}$ binding energy to $\mathrm{IrO}_{2}$ cluster by B3LYP and PBE. All the coordinates of the structures in Figure 2 and Figure 5 are provided in a separate file.

The Supporting Information is available free of charge on the ACS Publications website.

\section{AUTHOR INFORMATION}

\section{Corresponding Authors}

yuanping@ucsc.edu; wag@,wag.caltech.edu

The authors declare no competing financial interest

\section{ACKNOWLEDGMENT}

We thank Dr. Ravishankar Sundararaman, Dr. Hai Xiao, Dr. Tao Cheng and Dr. Yan Choi Lam for useful discussions. This paper is based on work performed in Joint Center for artificial photosynthesis - a DOE innovation hub, supported through the Office of Science of the U.S. Department of Energy under Award Number DE-SC0004993. We thank the National Energy Research Scientific Computing Center (NERSC) for providing computer time allocations.

\section{REFERENCES}

(1) Walter, M. G.; Warren, E. L.; McKone, J. R.; Boettcher, S. W.; Mi, Q. X.; Santori, E. A.; Lewis, N. S. Chem Rev 2010, 110, 6446. (2) Ma, T. Y., Cao J. L., Jaroniec M., Qiao S. Z., Angew. Chem. Int. Ed. 2016, 55, 1138 .
(3) Jiao, Y., Zheng, Y., Jaroniec, M., Qiao S. Z., Chem. Soc. Rev., 2015, 44, 2060

(4) Kumar, B.; Llorente, M.; Froehlich, J.; Dang, T.; Sathrum, A.; Kubiak, C. P. Annu Rev Phys Chem 2012, 63, 541.

(5) Rossmeisl, J.; Qu, Z. W.; Zhu, H.; Kroes, G. J.; Norskov, J. K. J Electroanal Chem 2007, 607, 83.

(6) Rossmeisl, J.; Logadottir, A.; Norskov, J. K. Chem Phys 2005, 319, 178.

(7) Man, I. C.; Su, H. Y.; Calle-Vallejo, F.; Hansen, H. A.; Martinez, J. I.; Inoglu, N. G.; Kitchin, J.; Jaramillo, T. F.; Norskov, J. K.; Rossmeisı, J. Chemcatchem 2011, 3, 1159.

(8) R. Sundararaman, W. A. Goddard III,, T. A. Arias, under preparation, 2016.

(9) Xiao, H.; Cheng, T.; Goddard, W. A.; Sundararaman, R. J Am Chem Soc 2016, 138, 483 .

(10) Spurgeon, J. M.; Velazquez, J. M.; McDowell, M. T. Phys Chem Chem Phys 2014, 16, 3623.

(11) Ping, Y.; Goddard, W. A.; Galli, G. A. J Am Chem Soc 2015, 137, 5264 .

(12) Garrity, K. F.; Bennett, J. W.; Rabe, K. M.; Vanderbilt, D. Comp Mater Sci 2014, 81, 446.

(13) Giannozzi, P. et al. J Phys-Condens Mat 2009, 21, 295502. Giannozzi, P.; Baroni, S.; Bonini, N.; Calandra, M.; Car, R.; Cavazzoni, C.; Ceresoli, D.; Chiarotti, G. L.; Cococcioni, M.; Dabo, I.; Dal Corso, A.; de Gironcoli, S.; Fabris, S.; Fratesi, G.; Gebauer, R.; Gerstmann, U.; Gougoussis, C.; Kokalj, A.; Lazzeri, M.; Martin-Samos, L.; Marzari, N.; Mauri, F.; Mazzarello, R.; Paolini, S.; Pasquarello, A.; Paulatto, L.; Sbraccia, C.; Scandolo, S.; Sclauzero, G.; Seitsonen, A. P.; Smogunov, A.; Umari, P.; Wentzcovitch, R. M. J. Phys.: Condens. Matter 2009, 21, 395502.

(14) Grimme, S. J Comput Chem 2006, 27, 1787.

(15) Henkelman, G.; Uberuaga, B. P.; Jonsson, H. J Chem Phys 2000, 113,9901

(16) Baroni, S.; de Gironcoli, S.; Dal Corso, A.; Giannozzi, P. Rev Mod Phys 2001, 73, 515.

(17) Bochevarov, A. D.; Harder, E.; Hughes, T. F.; Greenwood, J. R.; Braden, D. A.; Philipp, D. M.; Rinaldo, D.; Halls, M. D.; Zhang, J.; Friesner, R. A. Int J Quantum Chem 2013, 113, 2110.

(18) Fang, Y. H.; Liu, Z. P. J Am Chem Soc 2010, $132,18214$.

(19) Sundararaman, R.; Goddard, W. A. J Chem Phys 2015, 142, 064107.

(20) Ping, Y.; Sundararaman, R.; Goddard, W. A. Phys Chem Chem Phys 2015, 17, 30499.

(21) Letchworth-Weaver, K.; Arias, T. A. Phys Rev B 2012, 86, 075140

(22) Gunceler, D.; Letchworth-Weaver, K.; Sundararaman, R.; Schwarz, K. A.; Arias, T. A. Model Simul Mater Sc 2013, $21,074005$.

(23) Norskov, J. K., Rossmeisl, J., Logadottir, A. and Lindqvist, L. J. Phys. Chem. B 2004, 108, 17886-17892.

(24) McCrory, C. C. L.; Jung, S. H.; Peters, J. C.; Jaramillo, T. F. J Am Chem Soc 2013, 135, 16977.

(25) McCrory, C. C. L.; Jung, S.; Ferrer, I. M.; Chatman, S. M.; Peters, J. C.; Jaramillo, T. F. J Am Chem Soc 2015, 137, 4347.

(26) Ling, C.; Zhou, L. Q.; Jia, H. F. Rsc Adv 2014, 4, 24692.

(27) Zhang, P. X.; Dong, Y.; Kou, Y. L.; Yang, Z. Y.; Li, Y. P.; Sun, X. M. Catal Lett 2015, 145, 1169.

(28) Stoerzinger, K. A.; Qiao, L.; Biegalski, M. D.; Shao-Horn, Y. J Phys Chem Lett 2015, 6, 1548. 
